\title{
Consumo de cine extranjero en Monterrey
}

José Carlos Lozano Rendón*

Tecnológico de Monterrey - Monterrey

EL ESTUDIO sobre la oferta y consumo de mensajes extranjeros en América Latina en general, y en México en particular, ha experimentado un crecimiento considerable en los últimos años. De la posición simplista de muchos imperialistas culturales en los setenta de concluir desde los análisis económico políticos de los flujos la manipulación ideológica y la pérdida de identidad cultural, se ha pasado a una aproximación más cuidadosa, con investigaciones empíricas que documentan la oferta, la recepción y apropiación de dichos mensajes.

De entre todos los medios, el estudio de los flujos televisivos y su consumo por parte de las audiencias de distintas partes del mundo ha sido sin duda el más privilegiado. Tanto en Europa, como en Canadá, Asia y América Latina, diversos investigadores han estudiado las características de la oferta y los patrones de recepción en los países importadores.

En relación a la oferta de programación extranjera, investigaciones recientes muestran una tendencia hacia la disminución de las importaciones televisivas estadunidenses en muchos paises y una contraoferta de producciones locales y nacionales muy significativa (cfr. Biltereyst, 1992; Crovi, 1995; De la Garde, 1993; Lozano, 1995; Lozano y García Nuñez de Cáceres, 1996; Prado, 1992; Sepstrup, 1995; Straubhaar, 1993; Straubhaar et al., 1994; Straubhaar y Viscasillas, 1991). Esto no significa que la situación haya llegado a un nivel óptimo. De hecho, la ficción $-y$ hablar de ésta es hablar de la hecha en Estados Unidos-sigue siendo el género más extendido y popular en los sistemas televisivos de

* El autor agradece la valiosa colaboración de Rocío Fernández, Elsa García Nuñez de Cáceres, Adriana Garza, Iván López y Laura Tapia, de la Maestria en Comunicación del Tecnológico de Monterrey, Campus Monterrey. 
muchos países, incluyendo los desarrollados ( $c f r$. Biltereyst, 1992; Prado, 1992). Además, como aclara Straubhaar (1993), en la mayoría de los casos se trata de una interdependencia asimétrica, lejos aún del equilibrio deseable, y no se puede descartar el fenómeno del modelaje, es decir, la imitación de géneros, estructuras y contenidos extranjeros en las producciones locales. En el mejor de los casos, los hallazgos indican que existe una contraoferta de contenidos nacionales que abren la posibilidad para que los públicos tengan alternativas en su selección.

Los estudios de recepción, por su parte, muestran consistentemente una preferencia de los públicos de los países que importan programación estadunidense a los contenidos nacionales, cuando éstos existen ( $c f r$. Biltereyst, 1991; Caron y Bélanger, 1993; De la Garde, 1993; Martínez, 1992; Straubhaar y Viscasillas, 1991; Lozano, 1994, 1995; Lozano y García Nuñez de Cáceres, 1996) y lecturas negociadas u oposicionales hacia los contenidos extranjeros a los que sí se exponen (Ang, 1985; Fiske, 1987; Katzy Liebes, 1990). Estos últimos hallazgos, por lo general, han llevado a conclusiones muy optimistas por parte de algunos exponentes de los estudios culturales, quienes tienden a señalar que los mecanismos de resistencia y negociación de los públicos hacen muy difícil que se manifiesten efectos ideológicos negativos de los productos culturales extranjeros. Otros autores en esta misma corriente, sin embargo, son más cautelosos y exigen un mayor reconocimiento a los límites impuestos por las industrias culturales en las lecturas de los miembros de las audiencias y un análisis más profundo sobre el grado de actividad en los receptores (Biltereyst, 1991: 493; Morley, 1992: 39).

A pesar de que la investigación empírica sobre la oferta y el consumo de televisión extranjera se ha incrementado notablemente, como se advierte arriba, muchos autores se declaran insatisfechos con el estado actual de conocimientos e insisten en la necesidad de más trabajo en este rubro, especialmente a través de estudios cualitativos sobre la apropiación de los mensajes televisivos foráneos (cfr. Ang, 1990; Biltereyst, 1992).

Si lo anterior es válido para la investigación de la oferta y el consumo de televisión extranjera en el mundo, el cstado actual de la investigación sobre el cine es aún más limitado. Los pocos estudios existentes se han enfocado al fenómeno televisivo, descuidándose el análisis de la oferta de mensajes cinematográficos foráneos, asi como los procesos de recepción y apropiación de los mismos por los públicos de diferentes tipos de países. Si bien no se puede negar la importancia y trascendencia del fenómeno televisivo, los desarrollos tecnológicos y las transformaciones que ha sufrido en los últimos años la industria y el consumo de cine en el mundo justifican plenamente su 
estudio. Un dato que ilustra lo anterior es el proporcionado sobre las ganancias extranjeras de los estudios de Hollywood entre 1987 y 1992, las cuales se habían más que duplicado, esperándose que las ventas al exterior ( $42 \%$ de los ingresos totales en ese momento) sobrepasaran a las domésticas en los siguientes años (según datos de Business Mexico citados por Sánchez Ruiz, 1992: 190).

Podemos distinguir por lo menos tres razones por las cuales el cine mantiene una importancia indiscutible como medio de comunicación. En primer lugar, el surgimiento del videocaset y de establecimientos de renta de los mismos han facilitado el acceso masivo a una enorme cantidad de películas extranjeras que ya difícilmente se vuelven a encontrar en cartelera. A nivel mundial, por ejemplo, destaca la expansión de Blockbuster, la cual declaraba tener a fines de junio de 1996 3,348 establecimientos en Estados Unidos y 1,447 en el resto del mundo (Viacom, facts $\&$ figures, 1996). Dichos establecimientos ofrecen cada uno más de 5,000 títulos de películas, principalmente norteamericanas. En el caso de México, a mediados de 1995, Blockbuster declaraba contar con 100 videotiendas en México, y acaparar aproximadamente un 50\% del mercado nacional ("Inversión Blockbuster", 1995, julio). En segundo término, la expansión de la televisión de paga -primero a través del cable, luego mediante la señal restringida y más recientemente gracias a la televisión directa al hogar (DTH) - ha generado una enorme cantidad de canales basados en la programación de largometrajes, la mayoría de ellos estadunidenses. En tercer lugar, las salas de exhibición de largometrajes, más que desaparecer ante la actividad de los videoclubes y la televisión de paga, han sufrido un proceso de transformación hacia conglomerados de salas pequeñas (el concepto "multiplex" originado en Estados Unidos) ubicadas en centros comerciales (o cerca de ellos), con mayores atractivos en sus instalaciones (sonido, butacas, etc.). La vitalidad de la exhibición actual en dichas salas es clara si vemos la magnitud de las cifras que se recaudan en taquilla en numerosos países. En México, como ejemplo de la importancia de este medio de difusión, se calculaba que existían 1,434 salas en 1994 en todo el país ("Las 100 películas más taquilleras", 1995, septiembre-octubre).

A pesar de lo anterior, es muy poco lo que sabemos acerca de la oferta de películas extranjeras en los países importadores y menos aún de su consumo por parte de las audiencias.

Con respecto a lo primero, la oferta, la información disponible proviene principalmente de datos secundarios proporcionados por organismos gubernamentales y centros estadísticos, además de algunas investigaciones aisladas o que no tienen como propósito central analizar la oferta cinematográfica como tal (caso de los estudios sobre los géneros 
en la televisión). A continuación se presentan datos sobre la oferta de películas en las salas cinematográficas y a través de videoclubes y la programación televisiva.

La oferta de películas extranjeras en los 126 cines de la Ciudad de México y 1,344 de provincia (cfr. García Canclini, Holtz y Rosas Mantecón, 1984, p. 160) sobrepasa notoriamente a la de sus contrapartes nacionales. En 1990, el 52.6\% de los estrenos en el área metropolitana del Distrito Federal fueron películas estadunidenses, por un $20.5 \%$ de mexicanas según datos de la revista Dicine (Carro, 1991, mayo). En 1995, el 42.7\% de los estrenos eran de nacionalidad estadunidense y el 14.1\% nacional ${ }^{1}$ (Carro, 1996, mayo-junio). En ese mismo año, las 10 películas más exitosas en taquilla en México provenían de Hollywood (pp. 5-6). Según la revista Telemundo de las 100 películas más taquilleras en México en 1995, 75 eran norteamericanas, 10 mexicanas y el resto de otros países ("Las 100 películas más taquilleras", 1995, septiembre-octubre).

Sobre la transmisión de películas en los sistemas televisivos aéreos o de paga, investigaciones en diferentes partes del mundo muestran que es altamente significativa, ya que constituye uno de los elementos más importantes de lo clasificado bajo el género de "ficción". En la televisión abierta de la Ciudad de México, el género de la ficción ascendia en 1994 al 34\% del tiempo de la programación y un poco más de la mitad de este tiempo correspondía a películas. El 55\% de éstas últimas eran de nacionalidad norteamericana (Crovi, 1995: 184). En Guadalajara, Sánchez Ruiz (1994/95: 153) concluyó que las películas ocupaban un lugar privilegiado en las barras programáticas televisivas:

La agenda televisiva en Guadalajara incluye una dieta amplia de películas cinematográficas, en lo que no se diferencia mucho de la oferta de la ciudad de México [...]. De hecho, el "cine en televisión" ocupa el segundo lugar en términos de los diversos tipos y géneros de programas televisivos "ofertados", solamente después de los informativos.

Sánchez Ruiz agrega que del total de largometrajes transmitidos durante dos semanas en la televisión tapatía, $39 \%$ eran mexicanas y $53 \%$ extranjeras; $34.9 \%$ del total procedían de Estados Unidos. En el horario "triple A", el 33\% del tiempo lo ocupaban las películas, principalmente las de origen estadunidense. En la televisiónabierta de Monterrey, Lozano

1 El descenso en el procentaje de películas norteamericanas entre 1990 y 1995 quizás se deba a un cambio en la manera de clasificar los largometrajes eróticos. Mientras que en 1990 estos aparecían distribuidos según su nacionalidad, en 1995 ocuparon un lugar independiente en el conteo ( $25.6 \%$ del total), sin identificarse su origen. 
y García Nuñez de Cáceres (1996), encontraron que el 19.5\% del tiempo total de transmisión de los canales locales estaba ocupado por películas, y que el $78.9 \%$ de éstas procedían de Estados Unidos.

En cuanto a la oferta de cine extranjero a través de los 9,589 videoclubes registrados en México ( $c f r$. García Canclini, Holtz y Rosas Mantecón, 1984, p. 161), Sánchez Ruiz (1994/95: 159) detectó que en Guadalajara oscilaba entre el 70 y $80 \%$ de filmes estadunidenses en las grandes cadenas como Blockbuster, Videocentro y Multivideo. En los videoclubes independientes, con una cifra mucho menor de suscriptores, los porcentajes eran casi lo contrario: $80 \%$ de la oferta estaba constituida por largometrajes mexicanos contra un $20 \%$ de norteamericanos.

En términos generales, los autores concuerdan en señalar que a partir de la década de los ochenta, la asistencia a las salas de cine en México (y en muchas partes del mundo), ha descendido radicalmente. Getino (citado por García Canclini, 1994, p. 13) afirma que en el mayoría de los países de América Latina, durante la década pasada, "bajaron como promedio un 50 por ciento el número de salas y la asistencia de los espectadores". En México, la disminución fue dramática. Mientras que en 1984410 millones de personas asistieron a las salas cinematográficas, en 1991 sólo lo hicieron 170 millones (García Canclini, 1994, p. 14). Como aclara el propio García Canclini, esto no significa que no se vea cine, al contrario, hoy se ven más que en cualquier otra época gracias a la televisión y las videocaseteras.

Con respecto al consumo de películas extranjeras en México, hay algunos datos aislados que reflejan que a diferencia de lo que ocurre con los contenidos televisivos, en los cinematográficos prevalece el gusto por y consumo de los fóraneos, particularmente los estadunidenses. Sobre la exposición a películas extranjeras en las salas cinematográficas, Sánchez Ruiz (1994/95: 167) encontró que más de la mitad de su muestra de tapatíos prefería los extranjeros, mientras que sólo el $10 \%$ se inclinaba por las nacionales. En cuanto a géneros, el de acción era el más visto por los encuestados, seguido por el drama y, en tercer lugar, la comedia (pp. 166-167). En Monterrey, según el periódico El Norte, 16 de las 17 películas más taquilleras en el verano de 1995 fueron estadunidenses (Rosas, 1995, agosto 21). En Nuevo Laredo, Tamaulipas, una encuesta entre 575 alumnos de secundaria encontró que el $\mathbf{8 0 \%}$ de las películas vistas recientemente por ellos en salas cinematográficas provenian de Hollywood (Lozano, 1994: 233).

Sobre el consumo de películas en los hogares a través de la televisión, diversos estudios coinciden en señalar que es uno de los géneros más vistos. Según Sánchez Ruiz (1994/95: 154), la televisión es 
[...] el principal medio por el que el grueso de la población satisface sus deseos por ver películas cinematográficas. En prácticamente todas las investigaciones que conocemos, tanto a nivel nacional, como en diversas localidades de provincia y en el Distrito Federal, las películas son uno de los cinco principales tipos de programas televisivos preferidos (y atendidos) por el teleauditorio.

Una tercera parte de los encuestados por Sánchez Ruiz en Guadalajara, afirmó que prefería los filmes extranjeros, contra un $16 \%$ que declaró gustar de los nacionales (p. 179). Los hallazgos de Figueroa (1996: 88-92) sobre la programación vista por jóvenes de secundaria del Distrito Federal en la televisión coincide con lo anterior. Las películas ocupaban el cuarto lugar en las menciones de los alumnos, sin importar su clase social ni su sexo.

Muy similar era el caso para las películas vistas en video. Según datos del Ibope/México proporcionados por la revista $A d c e b r a$, casi un $62 \%$ de los telehogares mexicanos contaba en 1995 con videocasetera ("renace el cine", 1996, junio)². Según Sánchez Ruiz (1994/95: 174) el 85\% de los encuestados en Guadalajara declaró que la mayoría de los videos rentados por ellos procedían de Estados Unidos. La variable de escolaridad, según Sánchez Ruiz, era un buen predictor del tipo de gusto; a menor escolaridad mayor preferencia por las películas mexicanas (p. 179). En su investigación con jóvenes de secundaria de Nuevo Laredo, Tamaulipas, Lozano (1992: 87) encontró que el 87\% de las películas vistas recientemente con la videocasetera procedían de Estados Unidos. En forma similar a los hallazgos de Sánchez Ruiz, la clase social representaba un fuerte predictor para la preferencia de filmes por origen de los mismos: los de clase alta preferían los contenidos norteamericanos en una proporción significativamente mayor que los de clase media y clase baja (Lozano, 1992: 91). González (1994: 305), al realizar un análisis de la renta de videogramas en los dos videoclubes del poblado de Comala, Colima, encontró resultados contrapuestos, ya que en uno de ellos, 12 de los 15 títulos más rentados eran de nacionalidad norteamericana, mientras que en el segundo nueve videos de los 15 más rentados eran nacionales. Probablemente esto se explique con base en las

2 De acuerdo con estudios realizados por la oficina mexicana de la distribuidora de películas de la compañía Wait Disney, el número de videocaseteras en el pais asciende a los 7.2 millones (Aguilar, 1996, agosto 23). 
variables de nivel socioeconómico y educación de los suscriptores de uno y otro establecimientos, pero el autor no reporta datos al respecto.

Este incremento documentado de la exposición al cine a través de la televisión y la videocasetera ha despertado en los investigadores un interés por estudiar las diferencias en los procesos de apropiación en comparación con ver películas en las salas. García Canclini (1994, p. 17) al discutir las transformaciones del cine, se pregunta

¿Qué queda de todo esto cuando las películas se ven por televisión, en la sala iluminada de la casa, cortadas por anuncios publicitarios, por el llamado del teléfono o las intervenciones discordantes del resto de la familia? ¿En qué se convierte el cine cuando ya no se va al cine, sino al videoclub o se ve lo que azarosamente pasa la televisión? [...]

[...] ¿Hay otras formas de clandestinidad y fetichismo, de ritualidad y distinción, propias de los hábitos de consumo de cine en las pantallas chicas? ¿cómo se forma el saber filmico de las masas en la televisión y los videocentros, qué ha cambiado en los cinéfilos de sala que incorporan los nuevos recursos electrónicos para ver más cine? [...]

En síntesis, la mayoría de los hallazgos obtenidos en los estudios sobre oferta y consumo de cine extranjero, tanto en otros países como en México, difieren sustancialmente de los relativos a los flujos y la recepción de televisión extranjera. Por una parte, la contra oferta de películas nacionales es muy escasa, y por la otra, los receptores parecen preferir las producciones foráneas -especialmente las norteamericanas- sobre las locales. La exposición a filmes extranjeros -ya sea en salas, en canales televisivos o por medio de la videocasetera-parece ser muy alta, aunque claramente se observa que faltan más estudios empíricos que documenten los patrones de exposición y consumo en diferentes ciudades del país y en diferentes segmentos del público. Los pocos trabajos existentes sugieren que en el consumo de filmes estadunidenses influyen variables como la clase social, el sexo y el nivel educativo, aunque a estas tres variables se le podría agregar la de la edad, ya que hay razones para suponer que ésta también constituye una mediación, al igual que las anteriores.

La importancia de las mediaciones en la recepción de mensajes comunicacionales ha sido documentada ampliamente por numerosos teóricos e investigadores culturalistas (cfr. Downing, 1990; Fiske, 1987; Fuenzalida, 1989; Garcia Canclini, 1993; González, 1994, Martín Barbero, 1987, Orozco, 1991). Como señala Ang (1991: 160), las audiencias 
no son sólo consumidoras de mensajes, también son productoras de significado: "decodifican o interpretan los textos comunicacionales en formas relacionadas con sus circunstancias sociales y culturales y con la manera en que experimentan subjetivamente dichas circunstancias". Algunas de las mediaciones que intervienen en los procesos de recepción, precisamente, son las cuatro mencionadas arriba.

Con respecto a la variable sexo, Orozco (1991: 32) la ubica entre las mediaciones de "referencia" del sujeto. Para el investigador mexicano, ser "hombre" o "mujer" tiene implicaciones especificas en el proceso de la percepción en general y en el de la recepción en particular: "[...] Estudios recientes muestran cómo desde muy pequeños todos nosotros vamos desarrollando lo que se ha llamado el "patrón mental del género", que es precisamente eso, un patrón mental que influye la percepción masculina y femenina". Dichos patrones generan estereotipos de lo que es propio para un hombre y una mujer y esto, según Orozco, incide en los gustos y preferencias de uno y otro sexo. Estos estereotipos, a su vez, resultan "de la propia educación diferenciada de la que los adultos hemos sido objeto según hayamos nacido hombres o mujeres" (p. 32).

$\mathrm{El}$ estrato socioeconómico constituye asimismo una mediación de referencia que influye en los procesos de recepción. Aunque hay que evitar caer en una visión mecanicista adjudicándole a esta variable una total determinación en el consumo y apropiación de los mensajes comunicacionales, es indudable que establece parámetros y determina en cierta medida la disponibilidad de opciones culturales y reacciones a ellas (Morley, 1992: 55-57). Para Orozco (1991: 33-34) la pertenencia a un determinado estrato socioeconómico puede influir en la cantidad de programación que se consume diariamente, en el horario en que se puede o quiere ver televisión, en la manera en que se ve ésta (solo, acompañado) " $\mathrm{y}$, principalmente, en el tipo de programación que es consumida". Aunque estas afirmaciones se refieren al consumo televisivo, no parece advertirse algún obstáculo para trasladarlas a la recepción cinematográfica.

Aunque la edad no parece discutirse frecuentemente como mediación en los estudios culturales, parece válido tomarla como tal, e incluso relacionarla con la llamada por Orozco (1990/1991: 117-118) "mediación cognoscitiva". Aún queda mucho por explorar teóricamente sobre si la edad es mediación o reflejo, parte o dimensión de otra, pero si los hallazgos estadísticos presentan correlaciones, es importante detectarlas y analizar qué puede estar atrás de ello, como lo ha argumentado Morley en relación a la clase social (Morley, 1992: 56).

El presente trabajo se planteó como objetivo conocer las características del consumo de cine extranjero en Monterrey, tanto mediante la asistencia a salas cinematográficas 
como mediante la videocasetera. Asimismo, buscó explorar descriptivamente posibles relaciones entre dicho consumo y variables como sexo, edad y nivel educativo ${ }^{3}$.

Las preguntas de investigación que se formularon fueron las siguientes: 1) ¿Con qué frecuencia asisten los regiomontanos al cine y ven películas en videocasetera? 2) ¿Existe alguna diferencia por sexo, edad o nivel educativo en esa frecuencia de exposición? 3) ¿De qué nacionalidad son las películas que ven con mayor frecuencia? 4) ¿Existen diferencias por sexo, edad o nivel educativo en la preferencia de películas nacionales y extranjeras?

\section{Método}

El presente estudio se basó en la técnica de la encuesta telefónica por muestreo. La población que se tomó en cuenta fue aquella que tenía registrado su número en el directorio telefónico de 1995 del área metropolitana de Monterrey. Por lo tanto, quedaron excluidas de nuestra población aquellas personas que no contaban con servicio telefónico o cuyos números eran privados. De los números listados en el directorio telefónico se excluyeron todos aquellos que eran claramente comerciales y no residenciales.

Tomando como base un promedio de 392 números telefónicos particulares por página se calculó un total aproximado de 490,392. El tamaño de la muestra fue de 400 encuestas, de acuerdo al nivel de confianza del $95 \%$ y de un Error de Estimación del 5\%. El tipo de muestreo fue el polietápico, dadas las características de la base de la muestra. En la primera etapa se seleccionaron al azar 200 páginas de las 1,251 que cumplían las condiciones antes señaladas. En la segunda fase se eligió una de las cuatro columnas de cada página al azar, y en la tercera etapa, en cada una de las columnas asignadas, se seleccionaron ocho números al azar, dos para la muestra y seis para reemplazos.

La prueba piloto se realizó los días 29 de febrero y primero de marzo de 1996. El trabajo de campo se realizó entre el 5 y el 26 de marzo de 1996, con la participación de 17 alumnos de la Maestría en Comunicación del Tecnológico de Monterrey, Campus

3 Debido al diseño muestral de la presente investigación (encuestas por teléfono), que difícultaba la definición de la clase social de los participantes (además de excluir a los que no contaban con aparato telefónico), se decidió analizar el nivel educativo como una variable relacionada indirectamente con el estrato socioeconómico. 
Monterrey ${ }^{4}$. La selección del informante en cada vivienda se realizó en forma aleatoria, solicitando las iniciales de todos los habitantes de la misma mayores de 15 años y escogiendo uno de ellos al azar. En caso de que el elegido no estuviera en ese momento en casa, se hacía una cita y se volvía a llamar. Después de tres intentos fallidos, el número se reemplazaba por otro. La aplicación de cada encuesta tuvo una duración promedio de seis minutos.

Las principales variables del estudio fueron la exposición al cine extranjero (medida en asistencia mensual al cine y número de películas vistas por semana en la videocasetera), la nacionalidad de las películas vistas recientemente, la edad, el sexo y el nivel educativo. Esta última variable se preguntó en 12 rangos: primaria, primaria no terminada, secundaria, secundaria no terminada, preparatoria, preparatoria no terminada, técnica/comercial, técnica/comercial no terminada, profesional, profesional no terminada, estudios de postgrado, estudios de postgrado no terminados. Para las pruebas de análisis de varianza en una dirección, sin embargo, esta variable se recodificó en tres grupos solamente: hasta secundaria terminada, hasta preparatoria terminada y profesional o postgrado.

\section{Resultados}

El grado de exposición a las películas extranjeras en las salas cinematográficas y mediante la videocasetera por parte del público regiomontano se puede observar en los cuadros 1 y 2 . Cerca del $45 \%$ no acostumbraba asistir al cine y un $30 \%$ adicional iba solamente entre una y dos veces (véase el Cuadro 1). A diferencia de la televisión o de la videocasetera, la exposición a filmes en las salas cinematográficas era relativamente baja $^{5}$. Lo anterior, sin embargo, se matizaba por la mayor exposición de muchas de estas personas a películas en la televisión aérea y de paga (cfr. De la Garza, 1996; Díaz, 1995), así como a través de la videocasetera (Cuadro 2). De hecho, alrededor del $40 \%$ de los regiomontanos encuestados acostumbraban ver más de 2 películas por semana mediante

4. El diseño muestral y del cuestionario, asi como la organización y supervisión del trabajo de campo estuvo a cargo de Rocío Fernández, Elsa García Nuñez de Cáceres, Adriana Garza, Iván López y Laura Tapia. Participaron como encuestadores Hilda Alanis, Yamiletl de la Garza, Mónica Espinosa, Ruth García, René Garza, Luis M. López del Puerto, Elvira Toba, Miriam Toribio, José Treviño, Jesús Vargas y Luz Ma. Velázquez. El coordinador general de la investigación fue José Carlos Lozano.

5. Esta cifra de poca asistencia al cine es muy similar a la reportada para la Ciudad de México (cfr. García Canclini, Hotz y Rosas, 1994), Guadalajara (cfr. Sánchez Ruiz, 1994) y Tijuana (cfr. Valenzuela, 1994). 
este último medio, lo que indica la intensidad del uso y la importancia en la difusión de filmes de dicha tecnología ${ }^{6}$.

Los cuadros 3 y 4 muestran que la variable sexo no se asociaba con una mayor o menor asistencia a las salas o uso de la videocasetera. Tanto los hombres como las mujeres acostumbraban exponerse a los filmes con la misma intensidad. Esto coincide con lo encontrado en Mérida ( $c f r$. Quintal y Reyes, 1994, p. 277) y Tijuana ( $c f r$. Valenzuela, 1994, p. 327) donde no se identificaron diferencias significativas entre la frecuencia con que hombres y mujeres acostumbraban asistir a las salas cinematográficas.

La edad, por su parte, parecía influir en la frecuencia con que se asistía al cine (a mayor edad menor asistencia), pero no en el número de películas vistas mediante la videocasetera por semana ( $c f r$. Cuadro 5). Esto indica la fuerte correlación entre la asistencia al cine y los jóvenes, lo que justifica una veta de análisis importante dadas las características ya mencionadas de este segmento poblacional. Este hallazgo es muy consistente con lo encontrado para las ciudades de México, Guadalajara, Tijuana y Mérida, donde las investigaciones coordinadas por Néstor García Canclini concluyeron que a medida que aumentaba la edad de los encuestados disminuía la frecuencia con que asistían a las salas (cfr. García Canclini, Hotz y Rosas, 1994; Sánchez Ruiz, 1994; Valenzuela, 1994, y Quintal y Reyes, 1994). En contraste, los adultos regiomontanos veían películas mediante la videocasetera tan frecuentemente como los jóvenes (véase el Cuadro 5).

El nivel educativo parecía ejercer una influencia contraria a lo anterior. Como se puede advertir en el Cuadro 6, los de mayor nivel educativo acudían a los cines con mayor frecuencia que los de menor educación. Esto también coincide con lo encontrado para la asistencia a las salas en la Ciudad de México (cfr. García Canclini, Hotz y Rosas, 1994). ¿Significa lo anterior que los más educados deciden ir al cine por un deseo explícito de experimentar el ritual de plena concentración, clandestinidad y fetichismo al que se refiere García Canclini? Solamente mediante estudios cualitativos del consumo y apropiación del cine se podría llegar a una respuesta clara. De nuevo, al igual que en el resto de las

6. Aunque la variable "renta de películas por semana" se midió de una manera muy distinta, las investigaciones realizadas en el proyecto de "Los nuevos espectadores" coordinado por García Canclini, parecen indicar que en Ciudad de México, Guadalajara, Tijuana y Mérida se renta un mayor número de títulos por semana que en Monterrey (cfr. García Canclini, Hotz y Rosas, 1994; Sánchez Ruiz, 1994; Valenzuela, 1994, y Quintal y Reyes, 1994). 
variables, en el uso de la videocasetera no se reflejaba ninguna diferencia por los años de estudio de las personas (cuadro 7).

De acuerdo a lo anterior, se puede advertir que mientras la asistencia al cine se relaciona con la presencia de ciertas características (menor edad, mayor nivel educativo), la videocasetera ha logrado homogenizar de alguna manera la frecuencia con que se ven filmes en el hogar. El hecho de que más personas vean películas en este medio que en las salas cinematográficas permite advertir el relevante papel que ha adquirido esta tecnología en la diseminación de los productos culturales cinematográficos y en la transformación de los hábitos de entretenimiento de los regiomontanos.

El cuadro 8 demuestra claramente la inmensa popularidad de los filmes norteamericanos entre el público del área metropolitana de Monterrey. El $64 \%$ de las películas mencionadas como vistas recientemente eran de procedencia estadunidense, contra sólo un $8 \%$ de producción nacional y un $1.3 \%$ de origen europeo (Cuadro 8 ). En contraste con su preferencia de programas televisivos nacionales sobre los foráneos, los regiomontanos tenían una abrumadora predilección por contenidos estadunidenses. Si en la exposición televisiva el problema de la posible asimilación de valores ideológicos ajenos a la ideosincracia nacional se ha visto lejano por la contra oferta de contenidos nacionales y la correspondiente preferencia de éstos por la mayoría del público, un caso muy diferente se presenta en el caso del consumo de cine en salas o mediante la videocasetera. Esto, sin embargo, no cra privativo de los regiomontanos. Los públicos de Ciudad de México, Guadalajara, Tijuana y Mérida presentaban una clara preferencia de los filmes norteamericanos sobre los nacionales y los demás extranjeros ( $c f r$. García Canclini, Hotz y Rosas, 1994; Sánchez Ruiz, 1994; Valenzuela, 1994, y Quintal y Reyes, 1994). De hecho, en Mérida, más del $90 \%$ de los entrevistados declaró rentar sobre todo películas norteamericanas (Quintal y Reyes, 1994, p. 286).

¿Quiénes prefieren mayormente el cine estadunidense, los hombres o las mujeres? ¿los jóvenes o los adultos? ¿los de menor o mayor nivel educativo? Los cuadros 9 y $10^{7}$ proporcionan datos contradictorios sobre la influencia de la variable sexo. Mientras que en el primero se observa una ligera tendencia (no sigiifincativa en términos estadísticos)

7. En el cuestionario se le preguntó a los encuestados por los títulos de las dos últimas películas vistas recientemente en el cine o mediante la videocasetera. Las películas se reclasificaron por su procedencia y los resultados se cruzaron con las variables de sexo, edad y nivel educativo. Para cada variable se calcularon dos cuadros: uno para los títulos mencionados en primer lugar y otro para los mencionados en segundo lugar. 
a un mayor consumo de filmes norteamericanos por los hombres, en el segundo la diferencia (ésta sí estadísticamente significativa) se da en el sentido contrario. Estos datos se contraponen con los encontrados para la preferencia de programas televisivos por Lozano (1994) para los jóvenes de Nuevo Laredo, los de Olvera para los de Zacatecas (1995), los de Figueroa para los jóvenes de la Ciudad de México (1996), los de Díaz sobre los jóvenes de Monterrey (1995) y los de Valenzuela sobre el público de Tijuana (1994). En todos estos estudios, se observa con claridad una mayor preferencia de programación televisiva norteamericana por los hombres. En el caso del consumo de cine, sin embargo, Sánchez Ruiz (1994), reporta no haber encontrado diferencias en las preferencias cinematográficas estadunidenses entre los hombres y mujeres de Guadalajara incluidos en su muestra. ¿Significa esto que la influencia de la variable género en las preferencias sobre el origen de la programación televisiva no se reproduce de igual forma en las preferencias cinematográficas? Los datos de Lozano y Sánchez Ruiz sugieren que no, pero se requieren mayores estudios para validar estas posturas.

La influencia de la edad en la procedencia de las cintas, en contraste, se veía claramente definida. Los cuadros 11 y 12 muestran que a menor edad, mayor era el número de los títulos cinematográficos mencionados que provenían de Estados Unidos. Los jóvenes consumían una mayor cantidad de filmes de ese país en comparación con los adultos del área metropolitana de Monterrey, lo que de nuevo resalta la importancia que puede tener el cine norteamericano para este segmento poblacional. Este hallazgo no es consistente con los de Sánchez Ruiz (1994, p. 250) y Valenzuela $(1994$, p. 328) para los públicos de Guadalajara y Tijuana respectivamente, los cuáles no mostraban diferencias significativas en su preferencia de películas extranjeras por edad.

El nivel educativo también parecía ejercer cierto impacto en la preferencia por películas estadunidenses. En el cruce con los primeros títulos mencionados (Cuadro 13) la significancia estadística de la $X^{2}$ es muy alta ( $\left.p .=.0003\right)$, señalando que a mayor nivel educativo menor preferencia por el cine nacional y mayor por el norteamericano. Este hallazgo es similar al encontrado para las variables de nivel socio-económico y preferencias televisivas en diferentes estudios y ciudades del país: Monterrey, Irapuato, Ciudad de México, Nuevo Laredo, Zacatecas y Guadalajara ( $c f r$. Díaz, 1995; Blanco, 1996; Figueroa, 1996; Lozano, 1994; y Olvera, 1995; Sánchez Ruiz, 1994) y sugiere que las personas de menores ingresos o educación son lo que menos expuestos se encuentran a los contenidos y valores ideológicos foráneos. El hecho de que en el segundo cruce la prueba de la $\mathrm{X}^{2}$ no haya resultado significativa $(\mathrm{p} .=3290)$, sugiere cautela en las 
conclusiones anteriores aunque los datos continúan marcando una tendencia similar (véase Cuadro 14).

\section{Cuadro 1}

Asistencia mensual al cine entre los regiomontanos: 1996

\begin{tabular}{|c|c|c|}
\hline Veces al mes & Frecuencia & Porcentaje \\
\hline 0.0 & 170 & 45 \\
\hline $1.0-1.5$ & 116 & 30 \\
\hline $2.0-2.5$ & 51 & 14 \\
\hline $3.0-3.5$ & 24 & 6 \\
\hline $4.0-4.5$ & 15 & 4 \\
\hline $5.0-5.5$ & 5 & 1 \\
\hline Total & 381 & $100 \%$ \\
\hline
\end{tabular}

\section{Cuadro 2}

Películas vistas en videocasetera por semana

\begin{tabular}{|c|c|c|}
\hline Películas por semana & Frecuencia & Porcentaje \\
\hline 0.0 & 115 & 31 \\
\hline 0.5 & 3 & 1 \\
\hline $1.0-1.5$ & 104 & 28 \\
\hline $2.0-2.5$ & 76 & 20 \\
\hline $3.0-3.5$ & 48 & 13 \\
\hline $4.0-4.5$ & 14 & 4 \\
\hline $5.0-5.5$ & 11 & 3 \\
\hline 6.00 & 1 & 0 \\
\hline Total & 372 & $100 \%$ \\
\hline
\end{tabular}




\section{Cuadro 3}

Prueba t de películas vistas en el cine por sexo de los entrevistados

\begin{tabular}{|l|c|c|c|c|c|}
\hline Sexo & f & Media & Desv.Est. & $t$ & p. \\
\hline Femenino & 235 & 1.22 & 1.31 & 0.52 & .6000 \\
\hline Masculino & 136 & 1.30 & 1.44 & & \\
\hline Total & 371 & & & & \\
\hline
\end{tabular}

\section{Cuadro 4}

Prueba t de películas vistas por semana en la videocasetera por sexo de los entrevistados

\begin{tabular}{|l|c|c|c|c|c|}
\hline Sexo & f & Media & Desv.Est. & $t$ & p. \\
\hline Femenino & 229 & 1.61 & 1.47 & -0.04 & .9700 \\
\hline Masculino & 133 & 1.61 & 1.28 & & \\
\hline Total & 362 & & & & \\
\hline
\end{tabular}

\section{Cuadro 5}

Coeficientes de correlación ( $\mathrm{r}$ de Pearson) de asistencia mensual al cine y películas vistas por semana en la videocasetera por edad

\begin{tabular}{|l|c|c|c|}
\hline Medio & r & f & p. \\
\hline Cine & -.3637 & 380 & .000 \\
\hline Video & -.0960 & 371 & .065 \\
\hline
\end{tabular}

\section{Cuadro 6}

Análisis de varianza en una dirección de asistencia mensual al cine por nivel educativo

\begin{tabular}{|l|c|c|c|c|c|}
\hline Estudios & f & Media & Desv.Est. & f & p. \\
\hline Hasta secundaria & 138 & $1.01^{\mathrm{a}}$ & 1.33 & 3.641 & .0272 \\
\hline Preparatoria & 113 & 1.30 & 1.35 & & \\
\hline Profesional & 126 & $1.44^{\mathrm{a}}$ & 1.35 & - & \\
\hline Total & 377 & 1.24 & 1.35 & & \\
\hline
\end{tabular}

a Las medias con este simbolo son significativamente diferentes (Procedimiento b de Tukey). 


\section{Cuadro 7}

Análisis de varianza en una dirección de películas vistas en videocasetera por nivel educativo

\begin{tabular}{|l|c|c|c|c|c|}
\hline Estudios & f & Media & Desv.Est. & f & p. \\
\hline Hasta secundaria & 135 & 1.63 & 1.39 & 1.86 & .1570 \\
\hline Preparatoria & 103 & 1.80 & 1.60 & & \\
\hline Profesional & 130 & 1.44 & 1.26 & & \\
\hline Total & 368 & 1.61 & 1.42 & & \\
\hline
\end{tabular}

\section{Cuadro 8}

Procedencia de las últimas películas vistas en el cine o con la videocasetera

\begin{tabular}{|l|c|c|}
\hline Procedencia & Frecuencia & Porcentaje \\
\hline Nacional & 60 & 8 \\
\hline Estados Unidos & 477 & 64 \\
\hline Europa & 10 & 1 \\
\hline No recuerda-no sabe & 200 & 27 \\
\hline Total & $747^{\mathrm{a}}$ & $100 \%$ \\
\hline
\end{tabular}

a Este total se refiere al número de menciones, no de entrevistados. Cada uno de estos pudo mencionar hasta dos películas.

\section{Cuadro 9}

Procedencia de las últimas películas vistas en el cine o con la videocasetera por sexo de los entrevistados (1)

\begin{tabular}{|l|c|c|c|}
\hline Procedencia & Femenino & Masculino & p. de $\mathrm{X}^{2}$ \\
\hline Nacional & $13 \%$ & $6 \%$ & .0742 \\
\hline Estados Unidos & 87 & 94 & \\
\hline Total & $\mathrm{n}=176$ & $\mathrm{n}=115$ &. \\
\hline
\end{tabular}




\section{Cuadro 10}

Procedencia de las últimas películas vistas en el cine o con la videocasetera por sexo de los entrevistados (2)

\begin{tabular}{|l|c|c|c|}
\hline Procedencia & Femenino & Masculino & p. de $\mathrm{X}^{2}$ \\
\hline Nacional & 9 & 18 & .0431 \\
\hline Estados Unidos & 91 & 82 & \\
\hline Total & $\mathrm{n}=149$ & $\mathrm{n}=85$ & \\
\hline
\end{tabular}

\section{Cuadro 11}

Análisis de varianza en una dirección de la procedencia de las películas vistas en el cine o con videocasetera por edad (1)

\begin{tabular}{|l|c|c|c|c|c|}
\hline Procedencia & $\mathrm{f}$ & Media & Desv.Est. & F & p. \\
\hline Nacional & 31 & $41.1^{\mathrm{b}}$ & 15.7 & 16.26 & .0000 \\
\hline Estados Unidos & 266 & $28.8^{\mathrm{b}}$ & 11.3 & & \\
\hline Europa & 3 & 32.7 & 6.7 & & \\
\hline No sabe & 79 & 37.6 & 15.2 & & \\
\hline Total & 379 & 31.6 & 13.3 & & \\
\hline
\end{tabular}

a En el cuestionario se preguntó sobre el nombre y la procedencia de las dos últimas películas vistas en el cine o con videocasetera. Este cuadro reporta el origen de las cintas mencionadas en segundo lugar.

b Las medias con este símbolo son significativamente diferentes (Procedimiento b de Tukey).

\section{Cuadro 12}

Análisis de varianza en una dirección de la procedencia de las películas vistas en el cine o con videocasetera por edad (2)

\begin{tabular}{|l|c|c|c|c|c|}
\hline Procedencia & f & Media & Desv.Est. & F & p. \\
\hline Nacional & 29 & 35.1 & 13.6 & 6.012 & .0005 \\
\hline Estados Unidos & 209 & $29.0^{\mathrm{b}}$ & 11.7 & 1 & \\
\hline Europa & 7 & 30.6 & 10.0 & & \\
\hline No sabe & 121 & $34.9^{6}$ & 14.9 & & \\
\hline Total & 366 & & & & \\
\hline
\end{tabular}

a En el cuestionario se preguntó sobre el nombre y la procedencia de las dos últimas películas vistas en el cine o con videocasetera. Este cuadro reporta el origen de las cintas mencionadas en segundo lugar.

b Las medias con este símbolo son significativamente diferentes (Procedimiento b de Tukey). 


\section{Cuadro 13}

Procedencia de las últimas películas vistas en el cine o con la videocasetera por nivel educativo de los entrevistados $(1)^{\text {a }}$

\begin{tabular}{|l|c|c|c|c|}
\hline Procedencia & $\begin{array}{c}\text { Hasta } \\
\text { secundaria }\end{array}$ & Preparatoria & Profesional & p. de $\mathrm{X}^{2}$ \\
\hline Nacional & $20 \%$ & $8 \%$ & $4 \%$ & .0003 \\
\hline Estados Unidos & 80 & 92 & 96 & \\
\hline Total & $\mathrm{n}=99$ & $\mathrm{n}=86$ & $\mathrm{n}=113$ & \\
\hline
\end{tabular}

a En el cuestionario se preguntó sobre el nombre y la procedencia de las dos últimas películas vistas en el cine o con videocasetera. Este cuadro reporta el origen de las cintas mencionadas en primer lugar.

\section{Cuadro 14}

Procedencia de las últimas películas vistas en el cine o con la videocasetera por nivel educativo de los entrevistados $(2)^{a}$

\begin{tabular}{|l|c|c|c|c|}
\hline Procedencia & $\begin{array}{c}\text { Hasta } \\
\text { secundaria }\end{array}$ & Preparatoria & Profesional & p. de $\mathrm{X}^{2}$ \\
\hline Nacional & $15 \%$ & $14 \%$ & $8 \%$ & .3290 \\
\hline Estados Unidos & 85 & 86 & 92 & \\
\hline Total & $\mathrm{n}=79$ & $\mathrm{n}=73$ & $\mathrm{n}=87$ & \\
\hline
\end{tabular}

a En el cuestionario se preguntó sobre el nombre y la procedencia de las dos últimas películas vistas en el cine o con videocasetera. Este cuadro reporta el origen de las cintas mencionadas en segundo lugar.

\section{Discusión}

El presente trabajo se planteó como objetivo general conocer el consumo de cine extranjero en el área metropolitana de Monterrey, Nuevo León, México. Asimismo, buscó explorar relaciones entre dicho consumo y variables como el sexo, la edad y el nivel educativo.

En síntesis, los hallazgos de las 400 encuestas telefónicas sugieren que el consumo de cine extranjero -en especial del norteamericano- es muy superior al nacional, lo cual contrasta con lo que frecuentemente se detecta en la recepción televisiva. Esto sugiere que los interesados en analizar el impacto de la comunicación transnacional deberían 
prestar mayor atención a este medio, ya que presenta niveles de penetración mucho más significativos que los de los contenidos televisivos foráneos.

Asimismo, los resultados sugieren que el consumo de películas se da principalmente a través de la videocasetera y que la asistencia a las salas se relaciona principamente con los jóvenes y con un nivel educativo alto. Ni el sexo, ni la edad, ni el nivel educativo parecían asociarse significativamente con un mayor o menor consumo de películas en la videocasetera. Esta transformación del cine abre numerosas vetas de investigación relacionadas no sólo con la influencia ideológica de los contenidos cinematográficos, sino con la manera en que se ven y la forma en que las nuevas circunstancias de recepción propician apropiaciones diferenciadas.

Con respecto a las preferencias en la nacionalidad de los largometrajes, los datos indican que a menor edad y mayor nivel educativo era mayor la preferencia por las producciones estadunidenses. La influencia de la variable sexo, sin embargo, no se pudo detectar claramente, ya que mientras que con un bloque de películas preferidas no hubo diferencia significativa entre uno y otro sexo, en el segundo bloque apareció una mayor predilección de filmes extranjeros entre las mujeres.

Si bien la exposición a la televisión sigue siendo muy superior a la cinematográfica (en salas o mediante la videocasetera), esta última es suficientemente intensa como para ameritar estudios y análisis como el presente. Cerca del $68 \%$ de los encuestados veía una o más películas a la semana en la videocasetera y $55 \%$ asistía una o más veces al mes a salas cinematográficas. A estos datos habría que agregar el consumo de películas transmitidas en la televisión aérea o de paga, el cuál no fue cubierto en esta investigación. Llama la atención la importancia que ha adquirido la videocasetera como medio para consumir largometrajes, ya que ha facilitado que sin importar la edad, el sexo o el nivel educativo se consuma un mayor número de producciones cinematográficas.

La marcada preferencia por películas norteamericanas detectada en esta encuesta entre habitantes de Monterrey coincide con los hallazgos de otros estudios mexicanos en Ciudad de México, Comala, Colima, Guadalajara, Mérida, Nuevo Laredo y Tijuana. Si en el consumo televisivo se advierte una clara tendencia a preferir los contenidos nacionales, lo contrario ocurre en el consumo cinematográfico, abriendo asi la posibilidad de una mayor familiarización y asimilación de visiones del mundo y de la vida provenientes de Estados Unidos entre los diferentes públicos que se exponen a las cintas foráneas.

La naturaleza cuantitativa del estudio impidió analizar el funcionamiento de las variables sexo, edad y nivel educativo como mediaciones en la recepción y consumo de 
este tipo de mensajes. Por lo pronto, tratadas como variables, se observa en los resultados que por lo menos la edad y el nivel educativo sí manifiestan relaciones importantes con el consumo y la nacionalidad preferida de las películas. Estos dos factores constituyen sin duda elementos clave en la definición de las comunidades interpretativas, las cuales delimitan y determinan los procesos de interpretación de los mensajes comunicacionales (cfr. Lindlof, 1995). A ellos habría que agregar, como ha señalado García Canclini (1994), el análisis de la asistencia al cine no como acto individual, sino como "una compleja interacción social, un paseo con amigos, familiares o la pareja, con rituales, que requiere condiciones físicas y comunicacionales placenteras" (p. 337).

Las conclusiones que se obtengan del hallazgo sobre un alto consumo de películas norteamericanas deben de ser cautelosas. En primer lugar, el uso de una técnica cuantitativa como la encuesta circunscribió el proyecto al análisis de patrones de exposición y de preferencias, y no permitió profundizar en los procesos más complejos de apropiación, negociación y posible refuncionalización o rediseño de los contenidos cinematográficos. Podemos concluir que los regiomontanos ven mucho cine norteamericano, pero no sabemos hasta qué punto han asimilado los valores o visiones ideológicas que vienen en dichos mensajes, o de su capacidad para reelaborar y adaptar sus significados hegemónicos.

En segundo lugar, si los habitantes de esta ciudad se exponen principalmente al cine estadunidense, también es cierto que simultáneamente consumen más televisión nacional que extranjera. Los receptores no están expuestos ni consumen contenidos de un solo medio; en su vida cotidiana tienen contacto con una multiplicidad de mensajes y contenidos comunicacionales y es el conjunto de esa experiencia la que puede llegar a tener una determinada influencia en la ideología de los individuos.

En tercer término, hay que tomar en cuenta que, como afirma Bausinger (en Ang, 1991: 20), los medios no se usan completamente ni con total concentración: el grado de atención depende de la hora del día, los estados de ánimo, el lugar y la compañía en la que se da la exposición al medio: "los medios constituyen una parte integral de nuestras rutinas y rituales cotidianos. Por lo tanto, no se puede aislar el uso de los medios, puesto que está constantemente interrelacionado con otras actividades [...]". Esto resulta muy relevante ante el hallazgo de que hoy en día se consumen más películas mediante la videocasetera que mediante la asistencia a salas cinematográficas. El entorno doméstico en el que se ven las películas, asi, es cualitativamente diferente a la experiencia de la sala a oscuras que requierè y propicia una mayor concentración. ¿Cómo son descifrados los códigos y los significados del cine -pensado originalmente para ser consumido en la 
oscuridad de las salas cinematográficas, sin distracciones- por receptores "huidizos y solicitados por los múltiples ruidos perturbadores del hogar?" (Fuenzalida, 1989: 44-45).

En cuarto lugar, hay que preguntarnos si la abrumadora preferencia del cine norteamericano no responde en parte al sesgo de la oferta en las salas, los videocentros y los canales televisivos.

La investigación sobre el consumo y la apropiación de filmes extranjeros en México y América Latina es aún escasa y limitada. Se requieren tanto estudios cuantitativos que ofrezcan una panorámica general de los hábitos y preferencias así como estudios cualitativos que profundicen en los procesos de asimilación y negociación de los contenidos. En el contexto de la preocupación por el consumo de mensajes extranjeros y su impacto en la identidad cultural, los análisis de la oferta y consumo de películas parecen tener incluso más pertinencia que los de los programas televisivos, dada la preferencia de los contenidos nacionales en este último medio.

\section{Referencias bibliográficas y bibliografía}

AGUILAR, Alberto. (1996, agosto 23). Buena Vista Home, potencial. El Norte, p. 27A. ANG, Ien. (1985). Watching Dallas: Soap Opera and the melodramatic imagination. Londres, Methuen.

ANG, Ien. (1990). Culture and communication: towards an Ethnographic critique on media consumption in the transnational media system. European Journal of Communication, 5, 239-260.

ANG, Ien. (1991). The nature of the audience. En John Downing, Ali Mohammadi y Annabelle Sreberny-Mohammadi (Eds.) Questioning the media: a critical introduction. Newbury Park, Sage.

BILTEREYST, Daniël. (1991). Resisting American hegemony: a comparative analysis of the reception of domestic and US fiction. European Journal of Communication, 6, 469-497.

BILTEREYST, Daniël. (1992). Language and culture as ultimate barriers? an analysis of the circulation, consumption and popularity of fiction in small European countries. European Journal of Communication, 7, 517-540.

BLANCO, Jorge. (1996). Oferta de programas nacionales y extranjeros en medios televisivos y análisis de su exposición y consumo por alumnos de secundaria en la 
ciudad de Irapuato. Tesis de maestría, Tecnológico de Monterrey, Campus Eugenio Garza Sada (Monterrey).

Crovi, Delia. (1995). Entretener y vender, ¿fatal destino de la televisión mexicana? En José Carlos Lozano (Ed.) Anuario de Investigación de la Comunicación CONEICC II (pp. 177-198). Guadalajara: Consejo Nacional para la Enseñanza y la Investigación de las Ciencias de la Comunicación (CONEICC).

CARON, André y Pierre Bélanger. (1993). A reception study of American television products in Québec, en Roger de la Garde, William Gilsdorf e Ilja Wechselmann (eds.) Small nations, big neighbour: Denmark and Canada compare notes on American popular culture (pp. 133-150). Londres, John Libbey.

CARro, Nelson. (1991, mayo). 1990: un año de cine. Dicine, pp. 2-5.

CARro, Nelson. (1996, mayo). 1990: un año de cine. Dicine, pp. 2-5.

De la Garde, Roger. (1993). Dare we compare? En Roger de la Garde, William Gilsdorf e Ilja Wechselmann (Eds.) Small nations, big neighbour (pp. 25-64). Londres, John Libbey.

De la GaRZA, Yamille. (1996). Patrón de exposición y preferencias programáticas televisivas de los jóvenes de preparatoria de Monterrey y su área conurbada. Tesis de maestría, Tecnológico de Monterrey, Campus Monterrey.

DíAZ, Rocío. (1995). Oferta de mensajes televisivos extranjeros en Monterrey, N.L. y las preferencias programáticas de los jóvenes de secundaria. Tesis de maestría, Tecnológico de Monterrey, Campus Eugenio Garza Sada (Monterrey).

DownING, John. (1990). Preface. En J. Downing, A. Mohammadi y A. Sreberny (Eds.) Questioning the media: a critical introduction. Newbury Park, Calif., Sage.

FIGUEROA, Jaime. (1996). Oferta y consumo de programas televisivos extranjeros: un análisis de los alumnos de secundaria de la Ciudad de México. Tesis de maestría, Tecnológico de Monterrey, Campus Eugenio Garza Sada (Monterrey).

FISKE, John. (1987). Television culture. Londres, Methuen.

FUENZALIDA, Valerio. (1989). Visiones y ambiciones del televidente: estudios de recepción televisiva. Santiago de Chile, CENECA.

GARCía CANCLINI, Néstor. (1993). El consumo cultural y su estudio en México: una propuesta teórica. En N. García Canclini (Coord.) El consumo cultural en México. México, Conaculta.

GARCIACANCLINI, Néstor. (1994). Introducción: el cine mexicano en los noventa: ¿última crisis o crisis final?. En N. García Canclini ( Coord.) Los nuevos espectadores: cine, televisión y video en México (pp. 13-21). México, IMCINE/Conaculta. 
GARCía CANCLINI, Néstor, Holtz, Déborah y Rosas Mantecón, Ana. (1994). La Ciudad de México: cinéfilos y videófilos: ¿qué hay para ver? En N. García Canclini ( Coord.) Los nuevos espectadores: cine, televisión y video en México (pp. 159-227). México, IMCINE/Conaculta.

GoNZÁLEZ, Jorge. (1994). Más(+) Cultura(s). México, Conaculta.

HALL, Stuart. (1979). Encoding-decoding. En Stuart Hall, Dorothy Hobson, Andrew Lowe y Paul Willis (Eds.) Culture, media, language (pp. 128-138). Londres: Hutchinson.

Inversión Blockbuster. (1995, julio). Adcebra, p. 10.

KATZ, Elihu y Tamar Liebes. (1990). Interacting with Dallas: cross-cultural readings of American TV. Canadian Journal of Communication. 15, 45-65.

Las 100 películas más taquilleras de lo que va del 95. (1995, septiembre-octubre). Telemundo, pp. 30-32.

LINDLOF, Thomas. (1995). Qualitative communication research methods. Thousand Oaks, Sage.

LOZANO, José Carlos. (1992). Media exposure and cultural identity in a Mexican border community: the case of "Secundaria" students. Disertación doctoral, Universidad de Texas en Austin.

LozANo, José Carlos. (1994). Recepcióny uso de medios de comunicación en los jóvenes fronterizos. En José Carlos Lozano (Ed.) Anuario de Investigación de la Comunicación CONEICC II (pp. 217-246). Guadalajara, CONEICC.

LozANO, José Carlos. (1995). Oferta y recepción de televisión extranjera en México.

Comunicación y Sociedad, 25.

LoZANO, José Carlos. (1997). US. media reception in the Mexican Northern border. En Emile McAnany y Kenton T. Wilkinson (Eds.). Mass media and free trade: NAFTA and the cultural industries. Austin, The University of Texas Press.

LoZANO, José Carlos y Jorge García Nuñez de Cáceres. (1996). Oferta de programación televisiva en la televisión aérea del área metropolitana de Monterrey, Nuevo León. En Delia Crovi Druetta (Coord.) Desarrollo de las industrias audiovisuales en México y Canadá. México: Facultad de Ciencias Políticas y Sociales, UNAM.

MARTÍN BARBERO, Jesús. (1987). De los medios a las mediaciones: comunicación, cultura y hegemonia. México, Gustavo Gili.

MARTínez GarZA, Francisco J. (1992). Estudio de audiencias de televisión de Monterrey y su área metropolitana. Monterrey, N.L., Universidad Autónoma de Nuevo León. 
MORLEY, David. (1992). Television, audiences and cultural studies. Londres y Nueva York, Routledge.

OlVERA, Humberto. (1995). Análisis de la relación entre la oferta de programación nacional en medios televisivos y su recepción por alumnos de secundaria de la Ciudad de Zacatecas. Tesis de maestría, Tecnológico de Monterrey, Campus Eugenio Garza Sada (Monterrey).

OrozCO, Guillermo. (1990/91). La mediación en juego: televisión, cultura y audiencia. Comunicación y Sociedad, 10/11, 107-128.

OROZCO, Guillermo. (1991). Recepción televisiva: tres aproximaciones y una razón para su estudio. (Cuadernos de Comunicación y Prácticas Sociales No. 2). México, Universidad Iberoamericana.

PRADO, Emilio. (1992). Tendencias internacionales de programación televisiva. Telos, 31, 66-71.

PrehN, Ole y Else Jensen. (1993). Public service and the television marketplace. En Roger de la Garde, William Gilsdorf e Ilja Wechselmann (Eds.) Small nations, big neighbour: Denmark and Canada compare notes on American popular culture (pp. 217-235). Londres, John Libbey,

Quintal, Fanny y Reyes, Guadalupe. (1994). Mérida: ver cine en una ciudad de provincia. En N. García Canclini ( Coord.) Los nuevos espectadores: cine, televisión y video en México (pp. 262-297). México: IMCINE/Conaculta.

Renace el cine. (1996, junio). Adcebra, pp. 1, 48, 50, 53.

Rosas, Héctor. (1995, agosto 21). El Norte, p. 8E.

SÁNCHEZ RUIZ, Enrique. (1992). El espacio audiovisual mexicano ante el Acuerdo de Libre Comercio Canadá-Estados Unidos-México. Comunicación y Sociedad, 14/15, 177-197.

SÁNCHEZ RulZ, Enrique. (1994). Guadalajara: cine, televisión y video. En N. García Canclini ( Coord.) Los nuevos espectadores: cine, televisión y video en México (pp. 228-261). México, IMcINE/Conaculta.

SÁNCHEZ RUIZ, Enrique. (1994/95). Cine, televisión y video: hábitos de consumo fílmico en Guadalajara. Comunicación y Sociedad, 22/23, 147-184.

SEPSTRUP, Preben. (1995). Transnationalization of television in Europe. The Nordicom Review, 1.

STRAubHAAR, Joseph D. (1993). Más allá del imperialismo de los medios: interdependencia asimétrica y proximidad cultural. Comunicación y Sociedad, 18/19. 
STRAUBHAAR, Joseph y Gloria Viscasillas. (1991). Class, genre, and the regionalization of television programming in the Dominican Republic. Journal of Communication, 41.

StraubhaAR, J., S.M. Youn, C. Campbell, K. Champanie, L. Ha, S. Shrikhande, M. Elasmar, T.H. Ahn, M. C. Chen, S. Clarke, M. Takahashi (1994) "Mercados para la televisión regional y flujos de programas". Estudios sobre las Culturas Contemporáneas, 6 (18), 115-150.

VAlEnZUEla, Manuel. (1994). Tijuana: la recepción audiovisual en la frontera. En N. García Canclini (Coord.) Los nuevos espectadores: cine, televisión yvideo en México (pp. 298-329). México, IMCINE/Conaculta.

Viacom, facts \& figures. (1996). http://www.viacom.com/factsandfig/ 